\title{
Lemierre's syndrome: a rare cause of sepsis presenting with an absence of throat symptoms
}

\author{
Thomas Whittle, ${ }^{1}$ Natasha Amiraraghi, ${ }^{1}$ Bappa Sarkar ${ }^{2}$
}

${ }^{1}$ Ear,Nose and Throat Surgery, NHS Ayrshire and Arran, Crosshouse Hospital, Ayr, UK ${ }^{2}$ Radiology, NHS Ayrshire and Arran, Crosshouse Hospital, Ayr, UK

Correspondence to Dr Thomas Whittle, thomas.whittle.10@aberdeen. ac.uk

Accepted 7 April 2018

\section{SUMMARY}

A 16-year-old boy presented to hospital with a 6-day history of diarrhoea, vomiting and abdominal pain. During his admission he was found to be hypotensive, tachycardic and persistently feverish. Blood cultures taken on admission isolated Fusobacterium necrophorum. CT scanning of his neck showed a nonocclusive thrombus of the right internal jugular vein and a small right parapharyngeal abscess. CT scans of the chest and abdomen revealed multiple pulmonary abscesses, bilateral pleural effusions and splenomegaly. Treatment consisted of an unfractionated heparin infusion and intravenous antibiotics. A right-sided intercostal drain was inserted for a complex right-sided empyema. He subsequently developed a left-sided pleural effusion which was treated with a video-assisted thoracoscopic surgery (VATS) pleurodesis procedure. His fever resolved after his VATS pleurodesis procedure 3 weeks after initial presentation. Clinically he made a slow recovery but now is improved after 6 weeks of intravenous antibiotics and was discharged home.

\section{BACKGROUND}

Lemierre's syndrome is rare, characterised by internal jugular vein (IJV) thrombosis with septicaemia usually precipitated by oropharyngeal infection. ${ }^{12}$ Patients can then develop disseminated infective thromboembolic disease which can present in numerous ways; this presentation was particularly interesting as the patient had an absence of throat symptoms both at presentation and during admission. On retrospective questioning, the patient did recall a mild sore throat a week prior to admission.

Sore throat, neck pain and neck mass are the most common presenting complaints in Lemierre's syndrome. ${ }^{1}$ Gastrointestinal symptoms are not uncommon however are rarely recorded as sole presenting complaint. ${ }^{2}$ There are only few recorded cases of patients presenting with solely gastrointestinal symptoms in the absence of throat symptoms.

Lemierre's syndrome has become relatively rare since 1940s and the regular use of antibiotics for sore throat, one report even referring to it as the 'forgotten disease'. ${ }^{3}$ Despite this it is a diagnosis that the practicing otolaryngologist considers in some cases of throat sepsis.

On referral to secondary care, this patient had no throat symptoms and thus did not present to otolaryngology. This case helps to highlight Lemierre's disease as a differential in patients with evidence of sepsis without an obvious source. The case should be highlighted to a wide range of specialties within medicine given the possibility for unusual and multisystem presentations.

\section{CASE PRESENTATION}

A 16-year-old boy presented to hospital with a 6-day history of diarrhoea, vomiting, fever and upper abdominal pain. He denied any other symptoms on systemic enquiry.

On arrival he was feverish at $39.0^{\circ} \mathrm{C}$, tachycardic at 114 beats per minute, with a blood pressure of $105 / 65 \mathrm{~mm} \mathrm{Hg}$. His oxygen saturations were 99\% breathing room air and he was tachypnoeic with a respiratory rate of 24 breaths per minute. His ECG showed sinus tachycardia. An admission chest X-ray suggested a developing right-sided pleural effusion.

On examination, he was clinically dehydrated, with a clear sounding chest, normal heart sounds and a soft abdomen with generalised tenderness in left and right upper quadrants and epigastrium.

He had no medical history of note, took no regular medications and had no drug allergies.

The patient reported that other children at his school had a similar clinical picture recently.

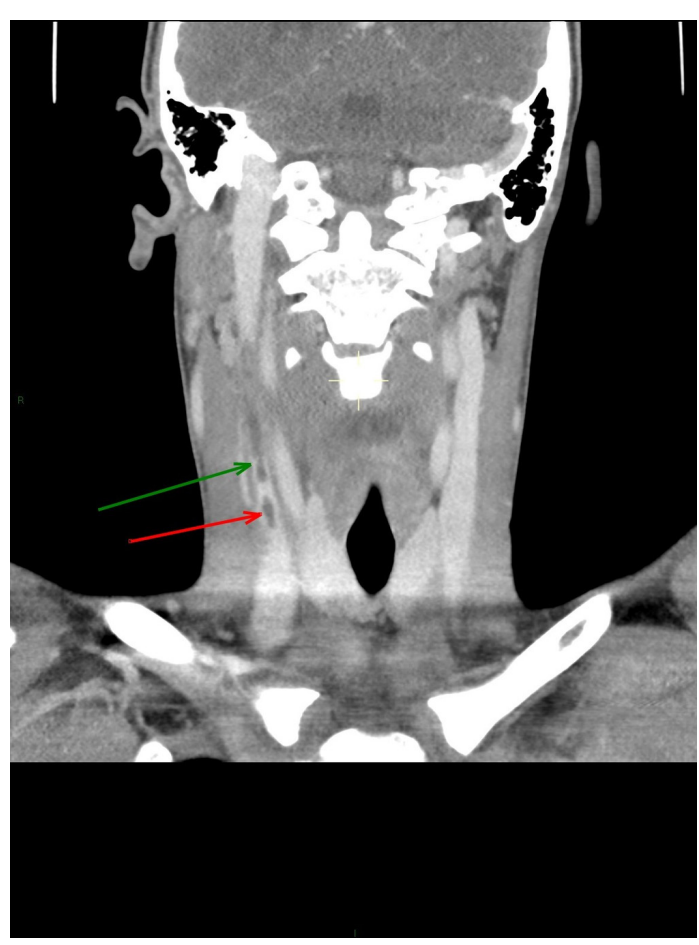

Figure 1 Coronal Ct scan showing right internal jugular vein thrombosis. 


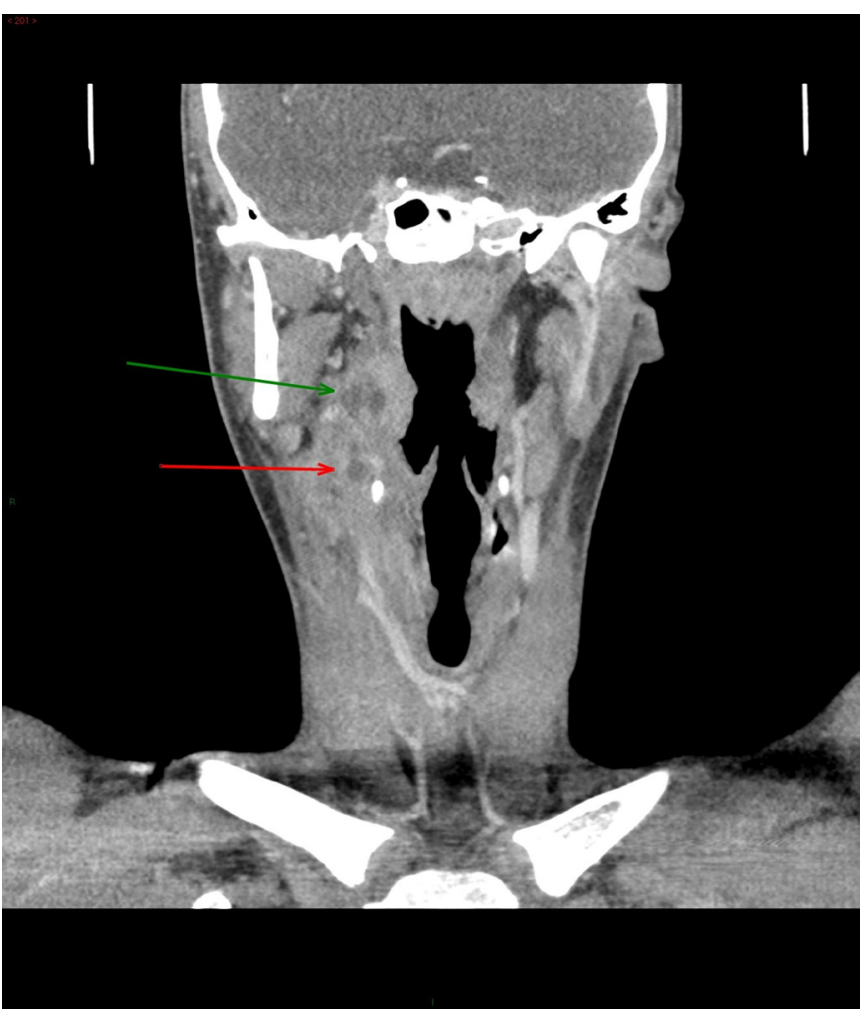

Figure 2 Coronal CT showing the right para pharyngeal abscess.

\section{INVESTIGATIONS}

Initial blood tests revealed raised inflammatory markers with $\mathrm{C}$ reactive protein of $195 \mathrm{mg} / \mathrm{L}$, leucocytosis of $15.0 \times 10^{9} / \mathrm{L}$ and platelet count of $51 \times 10^{9} / \mathrm{L}$. Liver function tests were also mildly deranged with bilirubin $51 \mu \mathrm{mol} / \mathrm{L}$, aspartate aminotransferase $130 \mathrm{U} / \mathrm{L}$ and alanine aminotransferase $92 \mathrm{U} / \mathrm{L}$. Renal function was impaired with uraemia of $11.6 \mathrm{mmol} / \mathrm{L}$, creatinine $102 \mu \mathrm{mol} / \mathrm{L}$, hyponatraemia $124 \mathrm{mmol} / \mathrm{L}$ and chloride $82 \mathrm{mmol} / \mathrm{L}$.

His blood film showed neutrophilia with slight left shift, toxic granulation and vacuolation and a genuine thrombocytopaenia.

Blood cultures taken on initial presentation grew a Fusobacterium necrophorum.

CT scanning of his neck, chest and abdomen revealed evidence of: right IJV thrombosis (figure 1 ) with a right parapharyngeal abscess measuring $10 \mathrm{~mm} \times 17 \mathrm{~mm} \times 20 \mathrm{~mm}$ (figure 2). There is evidence of bilateral evolving pulmonary abscesses (figure 3 ) with bilateral pleural effusions.

He subsequently underwent pleural aspiration of right-sided effusion which showed a hydrogen ion concentration of 55 and a lactate of 5.8 with lactate dehydrogenase of 3145 IU/L. This was treated as an empyema and he had a chest drain inserted.

\section{DIFFERENTIAL DIAGNOSIS}

Haemolytic uraemia syndrome was the initial differential diagnosis suspected due to the clinical history of infectious diarrhoeal symptoms with other children at school being affected and his biochemical blood picture. He was subsequently started on intravenous fluids replacement. Antibiotics were started for fever of unknown origin on day 3 of admission.

Due to the absence of throat sign's it is likely that Lemierre's syndrome would not have been suspected so early if it were not for F. necrophorum detected in blood cultures.

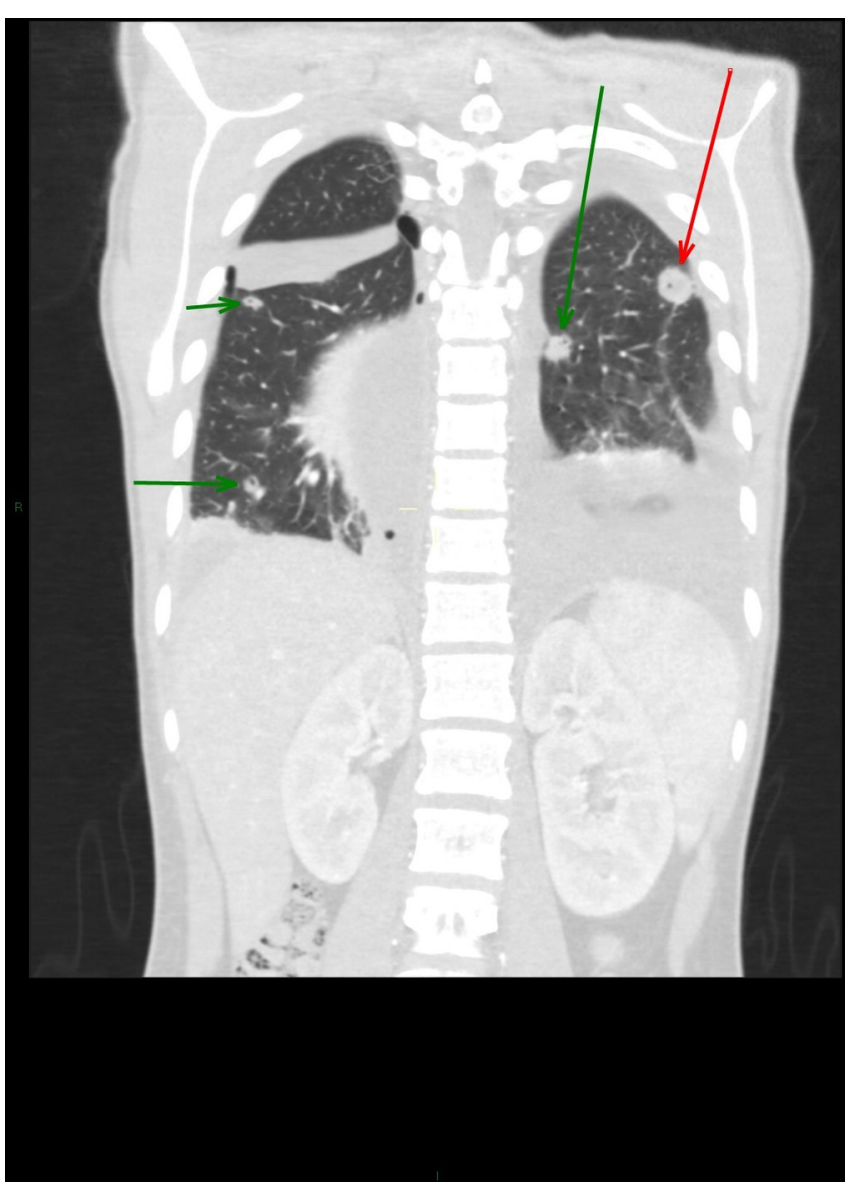

Figure 3 Coronal CT scan showing bilateral pulmonary abcesses.

\section{TREATMENT}

Treatment consisted of intravenous fluid replacement. Intravenous antibiotics, started on day 3 of admission, were gentamicin, amoxicillin and metronidazole. This was subsequently changed to $2.4 \mathrm{~g}$ of benzylpenicillin four times a day and metronidazole $500 \mathrm{mg}$ three times a day as per specialist microbiology advice after F. necrophorum was isolated from blood cultures.

An heparin infusion was commenced once the IJV thrombosis and septic emboli had been identified on CT scanning. This anticoagulation was subsequently stepped down to dalteparin 10000 Units daily.

During admission, the patient required insertion of a right intercostal drain for drainage of right-sided empyema. Unfortunately he continued to develop a large left-sided effusion which was treated with a - assisted thoracoscopic surgery (VATS) pleurodesis procedure by the cardiothoracic team.

An otolaryngology opinion was sought following the CT scanning findings of parapharyngeal abscess. Given the small size of the parapharyngeal collection, surgical drainage was deferred to intravenous antibiotic management in the first instance with a low threshold for surgical management should the abscess increase in size.

\section{OUTCOME AND FOLLOW-UP}

The patient was treated with intravenous antibiotics over a period of 6 weeks, his fever resolved on week 3 of his admission after the VATS pleurodesis procedure. He was discharged on oral antibiotics; amoxicillin ( $1 \mathrm{~g}$ three times a day) and metronidazole ( $400 \mathrm{mg}$ three times a day) to continue for a further 2 weeks. He was also discharged on rivaroxaban $15 \mathrm{mg}$ two times 
a day. Clinically he is much improved with no ongoing evidence of systemic infection.

$\mathrm{He}$ is to be reviewed as an outpatient following a repeat MRI scan of his neck and his chest with a view to assessing the right IJV thrombus and discontinuing his anticoagulation.

\section{DISCUSSION}

Lemierre's syndrome is defined by a pharyngeal infection, complicated by septicaemia and IJV thrombosis followed by septic emboli. ${ }^{3}$ Given the sequelae of the septic emboli, patients often require intensive care support. ${ }^{4}$ Lemierre's syndrome is known to affect a large age range of the population however the majority of cases occur between 13 and 40 years. ${ }^{5}$ Even when treated appropriately, mortality is reported to be around $2 \% .{ }^{6}$

Metastatic infective thromboembolic disease occurs commonly, with the reported incidence being $63 \%-100 \%{ }^{36}$ The lungs are the most common site but others include major joints, muscles and pericardium. As a result, the clinical presentation may vary greatly. ${ }^{3}$

Fever is the most common clinical finding in the patient with Lemierre's syndrome and is reported in 92\%-100\% patients, ${ }^{3}$ this is however non-specific and could potentially represent a number of pathologies. Pharyngitis, peritonsillar abscess and neck mass are the next most common findings and are more specific to Lemierre's syndrome with limited other differentials. An otolaryngologist will be suspicious of Lemierre's syndrome in patients with severe sepsis from tonsillitis or quinsy that is not responding to antibiotic therapy. It is very rare to have complete resolution of pharyngitis symptoms prior to symptoms from metastatic infection. ${ }^{6}$ This can mask the diagnosis. In our case report due to the absence pharyngitis symptoms and primary presentation with diarrhoea and vomiting, the diagnosis of Lemierre's syndrome was not initially clear and suspicions were raised only when the blood culture results were available.

The evidence for the role of anticoagulation in treatment of Lemierre's syndrome is inconclusive. ${ }^{6-8}$ In this disease, the risk of disseminated infection as a result of septic emboli particularly to the lungs is high. This can lead to cavitating lung lesions and empyema despite appropriate antibiotic treatment, as evidenced by this case. Anticoagulation treatment was therefore commenced in an attempt to stop the patient developing respiratory failure from further septic emboli. ${ }^{6}$ Some authors have suggested commencing anticoagulation therapy if little clinical improvement within 2-3 days of starting treatment or evidence of IJV thrombosis propagation. ${ }^{89}$ However due to limited research there are no set guidelines on the benefit of anticoagulation, when it should be commenced and the duration or type of treatment if commenced. Despite this it has been reported that as high as $64 \%$ of cases are treated with anticoagulation in a recent systematic analysis. ${ }^{6}$

In conclusion, this case demonstrates the wide variety of presentations of Lemierre's syndrome and the resultant difficulty with initial diagnosis. It is important that a wide range of subspecialty doctors should have knowledge of this condition.

\section{Learning points}

To have a low index of clinical suspicion for Lemierre's syndrome in an unwell patient presenting with upset gastrointestinal tract.

- Inclusion of Lemierre's disease in differential diagnosis in a patient with sepsis even in the absence of throat symptoms.

- To have a low threshold for neck imaging in a patient with sepsis if Fusobacterium necrophorum is isolated from blood cultures.

Contributors TW: clinical development fellow involved in the case and writing of the report at main author. NA: cochlear clinical fellow involved in the case management and assisted with writing of case report. BS: consultant radiologist involved in interpretation of radiology and acquisition of figures for the case report.

Funding The authors have not declared a specific grant for this research from any funding agency in the public, commercial or not-for-profit sectors.

Competing interests None declared.

Patient consent Parental/guardian consent obtained.

Provenance and peer review Not commissioned; externally peer reviewed.

(c) BMJ Publishing Group Ltd (unless otherwise stated in the text of the article) 2018. All rights reserved. No commercial use is permitted unless otherwise expressly granted.

\section{REFERENCES}

1 Karkos PD, Asrani S, Karkos CD, et al. Lemierre's syndrome: A systematic review. Laryngoscope 2009;119:1552-9.

2 Alperstein A, Fertig RM, Feldman M, et al. Septic thrombophlebitis of the internal jugular vein, a case of Lemierre's syndrome. Intractable Rare Dis Res 2017;6:137-40.

3 Eilbert W, Singla N. Lemierre's syndrome. Int J Emerg Med 2013;6:40.

4 Rae J, Misselbrook K. Lemierre's Syndrome - A rare cause of disseminated sepsis requiring multi-organ support. J Intensive Care Soc 2017;18:329-33.

5 Bank S, Jensen A, Nielsen HM, et al. Fusobacterium necrophorum findings in Denmark from 2010 to 2014 using data from the Danish microbiology database. APMIS 2016;124:1087-92.

6 6:Johannesen KM, Bodtger U. Lemierre's syndrome: current perspectives on diagnosis and management. Infect Drug Resist 2016;9:221-7.

7 Bondy P, Grant T. Lemierre's syndrome: what are the roles for anticoagulation and longterm antibiotic therapy? Ann Otol Rhinol Laryngol 2008;117:679-83.

8 Phua CK, Chadachan VM, Acharya R. Lemierre syndrome-should we anticoagulate? A case report and review of the literature. Int J Angiol 2013;22:137-42.

9 Lu MD, Vasavada Z, Tanner C. Lemierre syndrome following oropharyngeal infection: a case series. J Am Board Fam Med 2009;22:79-83.

Copyright 2018 BMJ Publishing Group. All rights reserved. For permission to reuse any of this content visit

http://group.bmj.com/group/rights-licensing/permissions.

BMJ Case Report Fellows may re-use this article for personal use and teaching without any further permission.

Become a Fellow of BMJ Case Reports today and you can:

- Submit as many cases as you like

- Enjoy fast sympathetic peer review and rapid publication of accepted articles

- Access all the published articles

- Re-use any of the published material for personal use and teaching without further permission

For information on Institutional Fellowships contact consortiasales@bmjgroup.com

Visit casereports.bmj.com for more articles like this and to become a Fellow 